\title{
Indicações de transplante de córnea no Hospital São Geraldo da Universidade Federal de Minas Gerais
}

\section{Indications for penetrating corneal graft at the São Geraldo Hospital of Minas Gerais Federal University}

Rafael Canhestro Neves ${ }^{1}$, Joel Edmur Boteon², Ana Paula de Morais Silva Santiago ${ }^{3}$

\section{Resumo}

Objetivo: Confrontar com a literatura os dados relacionados à idade, sexo e indicação dos transplantes penetrantes de córnea efetuados no Hospital São Geraldo da Universidade Federal de Minas Gerais (UFMG), de janeiro de 1999 a dezembro de 2005. Métodos: Realizou-se estudo retrospectivo de 887 ceratopatias penetrantes, com dados obtidos dos arquivos da Central Estadual de Notificação de Minas Gerais, MG Transplantes, e dos prontuários dos pacientes do Departamento de Córnea do Hospital São Geraldo. Os dados analisados foram:idade, sexo e diagnóstico pré-operatório das alterações corneanas. As indicações de transplante do período de janeiro de 1999 a junho de 2002 foram comparadas as de julho de 2002 a dezembro de 2005. Resultados: A média da idade dos pacientes foi de 48,3 anos, variando de 4 meses a 97 anos. As principais indicações de transplante penetrante de córnea foram: lesão ulcerativa (34,95\%), ceratocone $(16,35 \%)$, ceratopatia bolhosa do psedofácico $(16,01 \%)$, leucoma $(14,09 \%)$, falência primária (7,89\%), rejeição (5,64\%), distrofia endotelial de Fuchs (1,92\%), outras distrofias (1,47\%), causa indeterminada $(1,23 \%)$ e outras causas $(0,45 \%)$. Conclusão: A principal indicação de transplante foi para úlcera de córnea em vias de perfuração ou perfurada, responsável por 310 ceratopatias penetrantes. As indicações eletivas mais frequentes de transplantes foram para ceratocone, ceratopatia bolhosa do psedofácico e leucoma, com o crescimento das indicações para ceratopatia bolhosa do psedofácico no período. Mesmo com um aumento significativo no número de transplantes realizados, estratégias que busquem reduzir a alta incidência de ceratopatias penetrantes emergenciais decorrentes de úlceras corneanas devem ser implantadas.

Descritores: Doenças da córnea/cirurgia; Transplante de córnea; Ceratoplastia penetrante

\footnotetext{
'Aluno do Curso de Especialização em Oftalmologia do Centro Oftalmológico de Minas Gerais - Belo Horizonte (MG), Brasil; ${ }^{2}$ Doutor, Professor Associado do Departamento de Oftalmologia da Universidade Federal de Minas Gerais - FM/UFMG - Belo Horizonte - (MG), Brasil;

${ }^{3}$ Médica graduada pela Faculdade de Medicina da Universidade Federal de Minas Gerais - FM/UFMG - Belo Horizonte - (MG), Brasil.

Trabalho realizado no Serviço de Córnea e Doenças Externas do Hospital São Geraldo da Universidade Federal de Minas Gerais UFMG - Belo Horizonte (MG), Brasil.

Recebido para publicação em: 17/12/2009 - Aceito para publicação em 23/2/2010
} 


\section{INTRODUÇÃO}

$\mathbf{E}$ ntre os transplantes de órgãos e tecidos, o de córnea é o mais realizado no Brasil desde 1998, com aumento anual gradativo(1). Isto se deve a alguns fatores, como a ampliação da faixa etária da população, a melhor seleção de tecido doador, as novas técnicas operatórias que permitem a realização de cirurgias em situações consideradas inoperáveis até 10 anos atrás, a crescente conscientização da população, e pelo surgimento e atuação dos novos bancos de olhos no país ${ }^{(2)}$, do Sistema Nacional de Transplantes (SNT) através das Centrais Estaduais de Notificação, Captação e Distribuição de Órgãos (CNCDO's), e pelo interesse e apoio político.

No Brasil a principal indicação do transplante de córnea é o ceratocone. Contudo, há variações entre diferentes regiões e centros de referência. No Estado de São Paulo, responsável pelo maior número de ceratoplastias penetrantes (CPs) no país, o ceratocone ocupa a primeira posição, em Pernambuco a terceira ${ }^{(3-4)}$. Por outro lado, a prevalência de doenças corneanas também se altera entre os países, o que é atribuído às diferentes condições de saúde pública e níveis sócio-econômicos ${ }^{(2-18)}$.

$\mathrm{O}$ presente estudo tem como objetivo avaliar e divulgar as principais indicações de ceratoplastias penetrantes no Hospital São Geraldo (HSG) da Universidade Federal de Minas Gerais (UFMG), comparandoas com trabalhos da literatura científica.

\section{Métodos}

Realizou-se um estudo retrospectivo a partir do banco de dados da Central Estadual de Notificação, Captação e Distribuição de Órgãos de Minas Gerais - MG Transplantes - e dos prontuários correspondentes, nos quais foram avaliados 887 CPs no Setor de Córnea do HSG da UFMG no período de janeiro de 1999 a dezembro de 2005 , sendo avaliados a indicação cirúrgica e as características sexo e idade dos pacientes transplantados.

As indicações foram agrupadas em: lesões ulcerativas; ceratocone, ceratopatia bolhosa do pseudofácico (CBPF); leucoma (sem considerar suas causas); rejeição; falência primária; distrofia de Fuchs (DEF) e outras distrofias corneanas. Essa classificação foi proposta a fim de facilitar o cotejamento com dados da literatura de estudos similares.

O termo leucoma inclui sequelas de ceratite ou trauma. Lesões ulcerativas incluíram ceratites infecciosas ou não que estavam em atividade, com ameaça de perfuração ("melting" corneano e descemetocele) e úlcera perfurada. O diagnóstico de rejeição foi dado quando o edema do enxerto ocorreu após duas semanas de cirurgia, período este mínimo para reconhecimento do antígeno e desencadeamento do processo de rejeição ${ }^{(19)}$, diferenciando da falência primária, na qual ocorreu edema corneano no pós-operatório imediato, sem ceder ao tratamento por até 30 dias, demonstrando um provável comprometimento da córnea doadora ${ }^{(12,20,21)}$. Foi realizado o teste do qui-quadrado para verificação de possíveis diferenças entre frequências, utilizando $\mathrm{p}<0,05$ para rejeição da hipótese de nulidade.

Os diagnósticos para indicação das cirurgias foram classificados em percentual de incidência.

\section{$\underline{\text { Resultados }}$}

Dentre as 887 CPs realizadas no HSG/UFMG no período de Janeiro de 1999 a dezembro de 2005, 493 $(55,6 \%)$ eram do sexo masculino e $394(44,4 \%)$ do feminino (figura 1 ).

A idade destes pacientes variou de 0,4 até 97 anos, com média aritmética de 48,3 anos. As principais indicações para CPs (figura 2) foram: lesão ulcerativa em 310 $(34,95 \%)$, ceratocone em $145(16,35 \%)$, CBPF em 142 $(16,01 \%)$ e leucoma em 125 (14,09\%). Em menor frequência foram ainda observadas as indicações por falência primária em $70(7,89 \%)$, rejeição em 50 (5,64\%), DEF em 17 (1,92\%) e outras distrofias em 13 $(1,47 \%)$. Identificamos $11(1,23 \%)$ transplantes de pacientes que possuíam dados inconclusivos para o diagnóstico, classificados como de causas indeterminadas e 4 $(0,45 \%)$ que apresentaram outras causas não especificadas no presente estudo.

Trezentos e setenta e uma CPs $(41,8 \%)$ foram

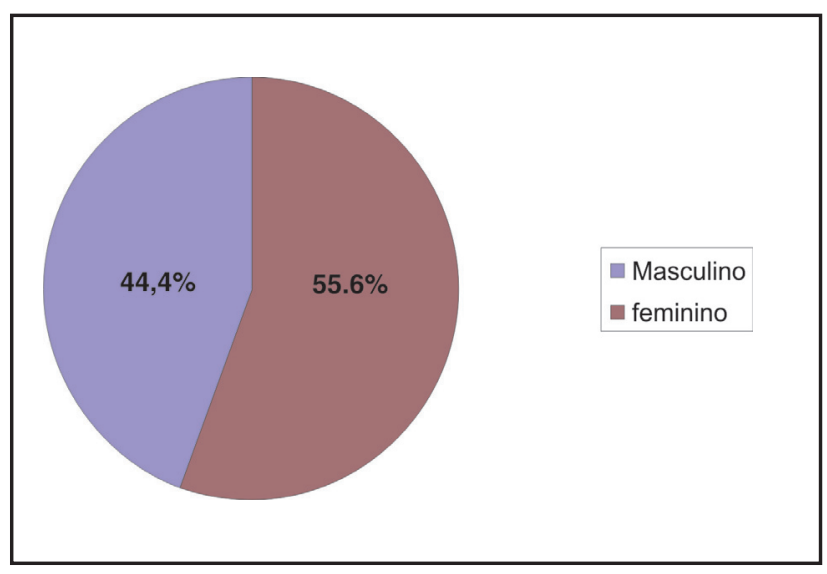

Figura 1: Distribuição percentual das ceratoplastias penetrantes realizadas no período de 1999 a 2005 no HSG/UFMG 


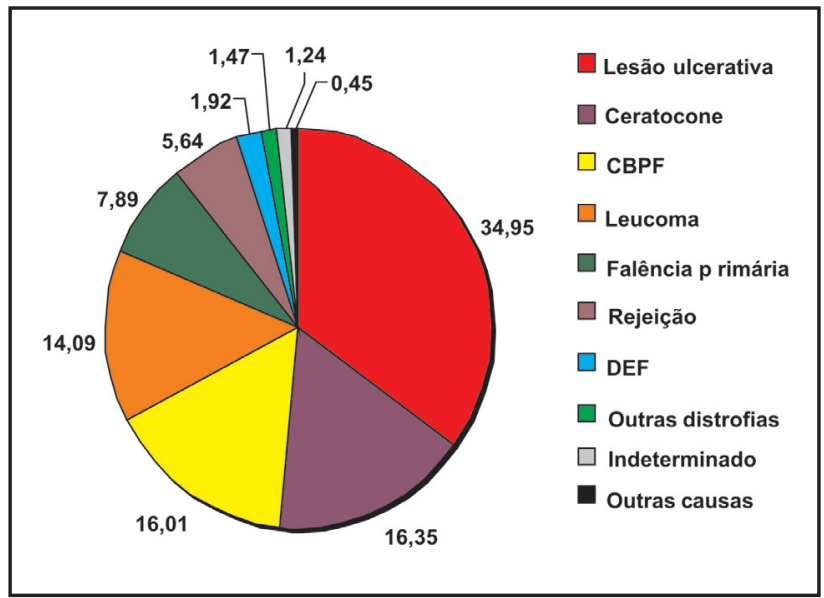

Figura 2: Distribuição percentual das indicações de ceratoplastia penetrante no período de 1999 a 2005 no HSG/UFMG

efetuadas no período de janeiro de 1999 a junho de 2002 e $516(58,2 \%)$ de julho 2002 a dezembro de 2005 (figura 3).

A figura 4 mostra a distribuição das indicações de CPs, permitindo a comparação do primeiro período, de 1999 a junho de 2002, com o segundo, de julho de 2002 a 2005.

A figura 4 evidenciou que nos dois períodos a lesão ulcerativa foi a principal indicação de CP nestes casos, $39,9 \%$ no primeiro período e $31,4 \%$ no segundo. Nas cirurgias eletivas observamos que a principal indicação no primeiro período foi ceratocone $(15,1 \%)$, ultrapassada no segundo período pela CBPF $(20,3 \%)$.

\section{Dıscussão}

Neste estudo foi observado discreto predomínio de CPs realizadas em pacientes do sexo masculino $(55,6 \%)$, o que ocorreu também no estudo realizado no mesmo hospital de 1983 a $1992^{(8)}$. Outros estudos realizados no país em centros de referência para este procedimento, ora são concordantes com o predomínio do sexo masculino $^{(3,6-17)}$, ora discordantes, com predomínio do feminino ${ }^{(10,11)}$, dependendo da região e da época de desenvolvimento do trabalho. Dados provenientes de outros países evidenciam esta mesma variabilidade ${ }^{(13-15,17)}$. O percentual maior do sexo masculino neste trabalho ocorre em parte pelo número de transplantes terapêuticos a que são expostos em nosso meio.

A média de idade dos pacientes desta amostra foi de 48,3 anos, quase 10 anos a mais do que a média obtida no mesmo serviço de 1983 a 1992, calculada em 39,2 $\operatorname{anos}^{(8)}$. Tal fato pode ter participação do envelhecimento da população brasileira com aumento constante da

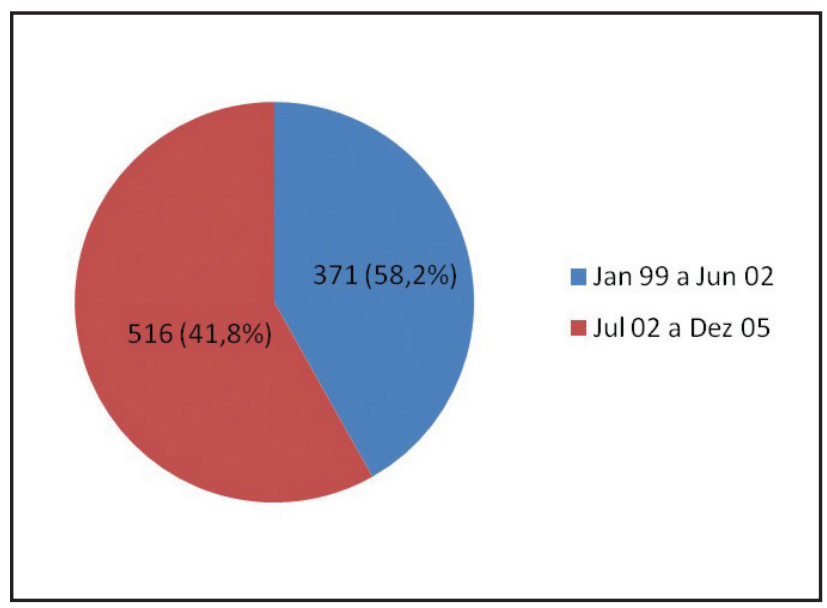

Figura 3: Número percentual de ceratoplastia penetrante, por período, realizados no HSG/UFMG

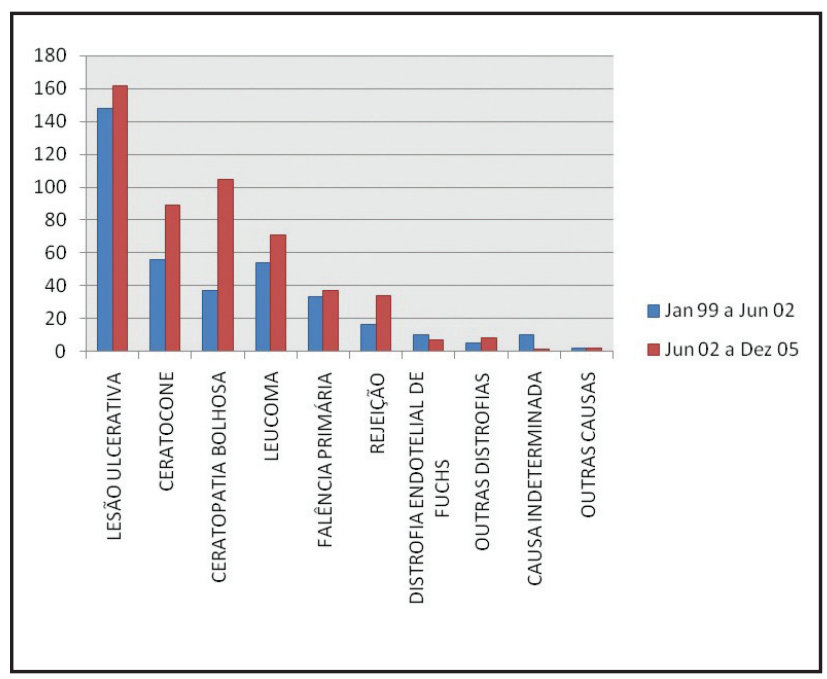

Figura 4: Distribuição das indicações absolutas de ceratoplastia penetrantes por período, no HSG/UFMG

expectativa de vida, do aumento da disponibilidade de córneas para transplantes, da conscientização das pessoas sobre as perspectivas atuais de tratamento e prevenção de doenças oculares, etc. Estudos desenvolvidos em outros hospitais brasileiros revelam médias de idade próximas às encontradas no presente estudo ${ }^{(3,7,10)}$. Países desenvolvidos evidenciam idades médias mais al$\operatorname{tas}^{(2,15,16)}$, uma vez que apresentam melhoras expressivas na expectativa de vida, no desenvolvimento técnico-científico, na informação e na prevenção.

O número de transplantes de córnea realizados no HSG/UFMG mostrou acentuada elevação. No período de 1983 a 1992 foram $184 \mathrm{CPs}^{(8)}$; de 1999 a junho de 2002 foram 371 casos e 516 de Julho 2002 a 2005. Este aumento expressivo pode ser atribuído à reestruturação do MG 
Transplantes e Banco de Olhos nesse período, somada a realização de campanhas educativas e de sensibilização da sociedade e a cooperação das autoridades e da mídia. Este novo cenário permitiu aumento e adequação da captação, cuidado e distribuição das córneas entre os centros que realizam este procedimento.

Com relação às principais indicações de CPs, as terapêuticas devido às lesões ulcerativas ocuparam o primeiro lugar (Figura 1) com 34,95\%, seguido pelas CPs eletivas, com o ceratocone (16,35\%) em segundo e CBPF $(16,01 \%)$ em terceiro. Contudo, a maioria dos estudos apresenta o ceratocone e a CBPF, procedimentos eletivos, como causas principais ${ }^{(6,7,11,14)}$. Atribuímos essa diferença em parte ao fato do HSG ser um centro de referência do Estado de Minas Gerais, recebendo a maioria dos casos emergenciais. Ressalta-se, contudo, a necessidade para uma abordagem precoce mais eficaz, através de uma melhor instrução dos profissionais e conscientização da população, visando uma redução do número de casos de CPs emergenciais, como já foi alcançado em outros estados e nos países desenvolvidos ${ }^{(2-12)}$.

Ao analisarmos as indicações eletivas, seguimos as tendências da maioria dos outros serviços com predomínio do ceratocone $(16,35 \%)$, CBPF $(16,01 \%)$ e leucoma $(14,09 \%)^{(3,5,19)}$ que não tiveram diferença significativa entre elas. O leucoma foi predominante sobre a falência primária $(7,89 \%)$ com $p<0,001$. Não se observou uma diferença significativa entre falência primária e rejeição $(5,64 \%)$, mas obtivemos novamente uma diferença significativa entre rejeição e DEF. Com isso, podemos observar nessa série uma distribuição classificatória das indicações eletivas da seguinte maneira: $1^{\circ}$ lugar: ceratocone, $\mathrm{CBPF}$ e leucoma; $2^{\circ}$ lugar: falência primária e rejeição; e $3^{\circ}$ lugar: distrofias corneanas (inclusive a de Fuchs).

A separação das indicações de ceratoplastia penetrante em dois períodos - 1999 a junho de 2002 e julho de 2002 a dezembro de 2005 - (figura 4) mostrou prevalência do ceratocone 56 casos $(15,1 \%)$ em relação à $\mathrm{CBPF}$ com 37 casos $(10,0 \%)$ no primeiro período. Já no segundo período, foram realizados 105 (20,3\%) transplantes devido a CBPF, que passou a ocupar após um crescimento expressivo o primeiro lugar, ficando o ceratocone em segundo com 89 casos (17,2\%). Atribuímos esse aumento vertiginoso da $\mathrm{CBPF}$ ao reflexo de um número crescente de pessoas que estão sendo submetidas à facectomia, espelho esse de múltiplas campanhas de conscientização, de uma técnica operatória moderna -facoemulsificação - e maior acessibilidade da população ao procedimento que tem como uma de suas principais complicações a CBPF.
Há a limitação temporal do presente estudo que abrangeu o período até 2005 , mas acreditamos que as tendências apresentadas se mantêm no serviço e para a verificação, outros estudos devem ser realizados.

\section{Conclusão}

Nesse estudo houve um crescimento do número de transplantes penetrantes de córnea no HSG com lesões ulcerativas como principal causa $(34,95 \%)$. A idade média dos pacientes operados no período de 1999 a 2005 aumentou em relação aos operados de 1983 a 1992. A indicação para ceratocone que ocupava a primeira posição no período de 1999 a junho de 2002, foi suplantada pela indicação de CBPF no período de julho de 2002 a dezembro de 2005. Estratégias que busquem a redução de lesões ulcerativas que evoluem para CPs devem ser implantadas, dado a sua alta incidência quando comparada com outros serviços.

\section{Abstract}

Purpose: To determine and compare with the literature data related on age, sex and the main indications for penetrating keratoplasty at the São Geraldo Hospital of Federal Universidade of Minas Gerais (UFMG), from january 1999 until december 2005. Methods: A retrospective analysis was performed using data from 887 patients submitted to corneal transplantation from january 1999 until december 2005. These data were obtained from the archives of the Central Notification of State of Minas Gerais, MG-Transplantes database, and from the medical records of the São Geraldo Hospital Corneal Department. The data collected were: age, gender and preoperative diagnosis of corneal diseases. A comparative analysis of the most common indications from january 1999 until june 2002 and from july 2002 until december 2005 was also performed. Results: The mean age of patients was 48.3 years, ranging from 4 months to 97 years. The most common corneal graft indications were ulcerative conditions $(34.95 \%)$, keratoconus (16.35\%), bullous keratopathy (16.01\%), leukoma $(14.09 \%)$, primary graft failure $(7.89 \%)$, graft rejection (5.64\%), Fuchs dystrophy (1.92\%), other dystrophies (1.47\%), undetermined causes (1.23) and other causes $(0.45 \%)$. Conclusion: The main indication for penetrating keratoplasty was corneal ulcer which was almost perfured or perfured, responding for 310 transplants (34.95\%). There was a substantial increase in the frequency of pseudophakic bullous keratopathy on 
the studied period. Even though, a significant increase in the number of corneal transplants, new strategies must be implanted to decrease the number of $P K$ for ulcerative conditions.

Keywords: Corneal diseases/surgery; Corneal graft; keratoplasty, penetrating

\section{RefERÊNCIAS}

1. Associação Brasileira de Transplante de Órgãos (ABTO). Registro Brasileiro de Transplantes - RBT [Internet]. [citado 2009 Jan 26]. Disponível em: <http://www.abto.org.br/abtov02/ $\mathrm{p}$ or t u g u e s/popula c a o/t ransplan te s/ estatisticasDeTransplantes.aspx?idCategoria $=5>$.

2. Lindquist TD, McGlothan JS, Rotkins WM, Chandler JW. Indications for penetrating keratoplasty: 1980-1988. Cornea. 1991;10(3):210-6.

3. Amaral CSR, Duarte JY, Silva PLS, Valbuena R, Cunha F. Indicações de ceratoplastia penetrante em Pernambuco. Arq Bras Oftalmol. 2005;68(5):635-7.

4. Calix Netto MJ, Giustina ED, Ramos GZ, Peccini RFC, Sobrinho M, Souza LB. Principais indicações de transplante penetrante de córnea em um serviço de referência no interior de São Paulo (Sorocaba - SP, Brasil). Arq Bras Oftalmol. 2006;69(5):661-4.

5. Cattani S, Kwitoko S, Kroeff MAH, Marinho D, Rymer S, Bocaccio FL. Indicações de transplante de córnea no Hospital de Clínicas de Porto Alegre. Arq Bras Oftalmol. 2002;65(1):95-8.

6. Fabris C, Corrêa ZMS, Marcon AS, Castro TN, Marcon IM, Pawlowski C. Estudo retrospectivo dos transplantes penetrantes de córnea da Santa Casa de Porto Alegre. Arq Bras Oftalmol. 2001;64(5):449-53.

7. Flores VGC, Dias HLR, Castro RS. Indicações para ceratoplastia penetrante no Hospital das Clínicas UNICAMP. Arq Bras Oftalmol. 2007;70(3):505-8.

8. Gonçalves EC, Trindade FC. Ceratoplastia penetrante: alterações nas indicações, 1983-1992. Arq Bras Oftalmol. 1994;57(4):274-7.

9. Dantas MCN, Dantas PEC, Holzchuh N, Lui Neto A, Giovedi Filho R, Giovedi M, Almeida GV. Indicações de transplante penetrante de córnea: 1991-1995. Arq Bras Oftalmol. 1998;61(1):26-33.

10. Sano FT, Dantas PEC, Silvino WR, Sanchez JZ, Sano RY, Adams F, Nishiwaki-Dantas MC. Tendência de mudança nas indicações de transplante penetrante de córnea. Arq Bras Oftalmol. 2008;71(3):400-4.
11. Endriss D, Cunha F, Ribeiro MP, Toscano J. Ceratoplastias penetrantes realizadas na Fundação Altino Ventura: revisão dos resultados e complicações. Arq Bras Oftalmol. 2003;66(3):273-7.

12. Eye Bank Association of America. Corneal transplant [Internet]. Washington, DC: EBAA; c2006 [cited 2010 Jan 26] Available from: <http://www.restoresight.org/general/ faqs.htm\#21>.

13. Tan DT, Janardhanan P, Zhou H, Chan YH, Htoon HM, Ang LP, Lim LS. Penetrating keratoplasty in Asian eyes: the Singapore Corneal Transplant Study. Ophthamology. 2008;115(6):975-82.

14. Xie L, Song Z, Zhao J, Shi W, Wang F. Indications for penetrating keratoplasty in north China. Cornea. 2007;26(9):1070-3.

15. Oliveira FC, Dantas PEC, Marco ES, Oliveira AC, NishiwakiDantas MC. Transplante terapêutico de córnea: resultados prolongados de série de casos. Arq Bras Oftalmol. 2007;70(4):625-31.

16. Dorrepaal SJ, Cao KY, Slomovic AR. Indications for penetrating keratoplasty in a tertiary referral centre in Canada, 1996-2004. Can J Ophthalmol. 2007;42(2):244-50.

17. Cosar CB, Sridhar MS, Cohen EJ, Held EL, Alvim Pde T, Rapuano CJ, et al. Indications for penetrating keratoplasty and associated procedures, 1996-2000. Cornea. 2002;21(2):148-51.

18. Teixeira MF, Almeida Júnior GC, Rodrigues ML, Kamimoto PS, Kashiwabuchi LK. Resultados e indicações de ceratoplastias penetrantes realizadas por médicos em treinamento, num país em desenvolvimento. Arq Bras Oftalmol. 2001;64(6):557-61.

19. Panda A, Vanathi M, Kumar A, Dash Y, Priya S. Corneal graft rejection. Surv Ophthalmol. 2007;52(4):375-96. Review.

20. Van Rensburg PD, Raber IM, Laibson PR, Eagle RC Jr. Management of primary corneal graft failure. Cornea. 1998;17(2):208-11.

21. Hirai FE, Klatte S, Pacini KM, Sato EH. Falência primária pós-transplante de córnea em serviço universitário. Arq Bras Oftalmol. 2002;65(6):655-7.

\section{Endereço para correspondência: \\ Rafael Canhestro Neves}

Rua São Paulo, no 2207/901 - Lourdes

CEP 30170-132 - Belo Horizonte (MG), Brasil

E-mail: rafacanhestro@yahoo.com.br 